\title{
La salud en el Perú en tiempos del coronavirus: Cinco meses después, entre el coronavirus, el gobierno, la corrupción y la ignorancia, ¿Sin escape?
}

\section{Health in Peru in the time of the coronavirus: Five months later, between coronavirus, government, corruption and ignorance, ¿Without escape?}

\section{Jesús Lorenzo Chirinos-Cáceres'}

Han pasado 163 días desde la llegada del primer caso al Perú y 152 días de iniciada la cuarentena y me atrevería a decir que la pandemia ha seguido su curso natural a pesar de todos los esfuerzos realizados, pero con muchos errores, y la grandiosa pérdida económica que hemos tenido.

Perú se ubica entre los países con peores cifras de contagios y fallecidos, en la actualidad: EEUU, Brasil, India, Rusia, Sudáfrica, Perú, México, Colombia, Chile e Irán y, acercándose nuevamente, España y Reino Unido. ${ }^{1}$ Perú, con cierta estabilidad económica pero no tan bueno o precario en salud y educación, está en el sexto lugar de países con más contagios (525 803 infectados, utilizando más pruebas rápidas que moleculares, y 26075 fallecidos, segundo lugar en mortalidad en el mundo), con 219 fallecidos en el día y tan solo 359781 recuperados, cifras de las que tenemos muchas dudas y que recién están sincerándolas, tanto las de infectados como las de fallecidos.

Veamos un ejemplo, si usamos pruebas rápidas y encontramos una persona con IgM positivo, podemos decir que se infectó hace una, dos o tres semanas, por lo tanto no es una infección nueva sino más bien prevalente y lo peor es que debemos decir que hace una, dos o tres semanas viene infectando, sin saberlo, de dos a cinco personas, dependiendo de la situación del número de infectados en la zona, Es decir, si esta persona interactúa en una zona de alta contagiosidad, y suponemos que hay muchos contagiados, la mayor probabilidad es que contagie a una o dos personas. Pero, si se moviliza a zonas de baja contagiosidad, contagiará de tres a cinco personas. Por lo que podríamos

Médico internista y salubrista. Facultad de Salud Pública, Universidad Peruana Cayetano Heredia, Lima, Perú.
Chirinos-Cáceres, J. La salud en el Perú en tiempos del coronavirus: Cinco meses después, entre el coronavirus, el gobierno, la corrupción y la ignorancia, ¿Sin escape?. Rev Soc Peru Med Interna. 201 9;33(3):96-97. https://doi.org/10.36393/spmi.v31i2.544

decir que la prueba sirve para analizar contagios ocurridos pero no para identificar al nuevo contagiado, tal que se haga un seguimiento a este y sus contactos, sin contar los falsos positivos o falsos negativos que puedan haber. Si hacemos pruebas moleculares, podemos tener infectados nuevos del último, penúltimo o antepenúltimo día y que vienen infectando también a una o dos personas en zonas de alta infección o tres a cinco de zonas de baja infección. De allí el valor insoslayable de las pruebas moleculares para identificar a los recién infectados y seguirlos a ellos y sus contactos. Esto debió de hacerse al inicio de la cuarentena o cuando se tuvo el primer caso. Luego de pasado todo este tiempo, la situación se ha tornado compleja y en cómo seguir los casos y los contactos con el número actual de infectados que tenemos a la fecha. El coronavirus nos acorraló, no hay escapatoria, nada más que protegerse con todas las medidas consabidas para no infectarnos.

La otra duda es la de los porcentajes de pruebas rápidas y moleculares realizadas. Si el porcentaje de pruebas rápidas es alto, lo más probable es que también tengamos entre ellos un gran número de recuperados entre la segunda y tercera semana, por lo que el número de recuperados es mucho mayor a lo que se señala; amén de los que están infectados, que no se han hecho las pruebas y están bien. Por tanto, ¿Para qué me sirven los números de los recuperados si solo son los hospitalizados? Esto descubre que hubo gente que debió hospitalizar pero pasó la enfermedad en su casa, obligada por las directivas del gobierno de esperar en casa a que lleguen los de las pruebas con su kit de "medicinas para el coronavirus" (sin evidencia de eficacia alguna, nada más que opiniones de algunos expertos) o porque no encontró cama. 
Nos dijeron al principio que el aislamiento social o la cuarentena era para romper la contagiosidad, luego con los "martillazos" se lograría aplanar el pico y llegar a una meseta que hasta ahora no la vemos; en cambio, hay un repunte de casos por la reapertura económica, el uso del transporte terrestre y aéreo y los bonos que ha dado el gobierno ocasionando aglomeraciones, donde no solo hubo exposición sino un tiempo prolongado de ello, y terminaron infectándose. ${ }^{2,3}$ Atrapados por el gobierno en nuestras casas, con un aislamiento social inicial por dos semanas, que ha tenido cuatro ampliaciones de cuarentena total y dos de cuarentena focalizada, encerrando sanos con niños, adolescentes y jóvenes potencialmente infectados ya que, con o sin prueba rápida o molecular, igual se infectaban los contactos con diferencia de semanas, como indicamos anteriormente.

Bien, todo lo anterior indica que, desde el punto de vista epidemiológico y estadístico, nosotros nos movemos entre lo observado, lo cual podemos medir en cierta forma si somos rigurosos con un más o menos margen de error, para aproximarnos a lo real. Observar las curvas que siguen en alza, a diferencia de algunos países que parece que alcanzaron mesetas, por lo que lo real en nuestro medio es que el coronavirus continúa su avance y que quizás ayuden los cercos epidemiológicos.

En consecuencia, todo lo señalado sobre tratamiento son observaciones o apreciaciones sin evidencia, que podría ayudar a paliar la pandemia, con un uso compasivo, hasta encontrar la vacuna o el tratamiento curativo, siendo este último todavía difícil de encontrar.

Los aspectos éticos también entran en juego cuando decidimos a quién le prescribimos un placebo o probamos hidroxicloroquina contra ivermectina, con la posibilidad de obtener consecuencias adversas. Lo peor de todo es cuando salen autoridades o gobernantes pugnando por uno $\mathrm{u}$ otro medicamento, señalando las bondades de los mismos o, mucho peor, aun cuando pregonan que encontraron la cura con el dióxido de cloro, por ejemplo.

La corrupción, además, en conseguir los equipos de protección personales, las mascarillas, las medicinas, el oxígeno, que para mí es vital -dado que si hay una saturación de oxígeno por debajo de $95 \%$ ya se debe conseguir un balón de oxígeno para utilizarlo en casa porque los hospitales están saturados y no hay camas en UCI, ventiladores, ni medicamentos esenciales para entrar a ventilación asistida- tornando la situación en compleja y dramática. Sin embargo, nos hemos dado el lujo de rechazar donaciones de oxígeno y las centrales simples de oxígeno que ofrecían algunas empresas privadas. El problema es que no hay presupuesto para gastos menores, solo para gastos mayores porque allí puede haber alguna ganancia non sancta. ${ }^{4,5}$ Veamos qué pasa con las vacunas.

Finalmente, la ignorancia -no es despectivo, ni insulto y, además, existe- de la población es madre de las creencias y de los abusos del poder. ${ }^{6}$ Veamos, esto es común no solo en nuestros países de ingreso bajo o medio sino también en los de altos ingresos. Podemos reconocer que puede ser una de las causas cuando grandes grupos de jóvenes o de edad intermedia hacen reuniones, fiestas e incitan a aglomeraciones sin medir el peligro de la alta contagiosidad y morbilidad-mortalidad de este COVID-19 y, en cierto modo, estimulados por el desatino en las medidas de algunas autoridades.

En esta parte del camino, sólo nos queda rezar y esperar por la vacuna, que deberá pasar por todos los controles de calidad antes que las ventajas políticas. Es una pandemia, no una situación aislada y como tal debemos enfrentarla.

\section{REFERENCIAS BIBLIOGRÁFICAS}

1. Coronavirus Resource Center. COVID-19 Map - Johns Hopkins University. URL disponible en: https://coronavirus.jhu.edu/map. html (Fecha de acceso:15 de agosto 2020).

2. Chirinos-Cáceres JL. COVID-19: La salud en el Perú en tiempos del coronavirus: Areflexionar. Rev Soc Peru Med Interna. 2019;33(1):1011. URL disponible en: https://doi.org/10.36393/spmi.v33i1.504

3. Chirinos-Cáceres, J. La salud en el Perú en tiempos del coronavirus: 60 días después, ¿sin reflexión? Rev Soc Peru Med Interna. 2019;33(2): 56-57. URL disponible en: https://doi.org/10.36393/ spmi.v33i2.520

4. García PJ. Lecture: Corruption in global health: the open secret. The Lancet/The Academic Medical Sciences. Published Online November 27, 2019. URL disponible en: https://doi.org/10.1016/ S0140-6736(19)32527-9 www.thelancet.com http://dx.doi. org/10.1016/S0140-6736(19)32527-9

5. Chirinos-Cáceres, JL. La corrupción en el sector salud: ¿A quién afecta? Rev Soc Peru Med Interna. 2019;32(4): 125-126. URL disponible en: https://doi.org/10.36393/spmi.v32i4.489.

6. Erasmo de Roterdam. Moriae Encomium, sive Stultitiae Laus. Elogio de la locura. URL disponible en: https://es.wikipedia.org/ wiki/Elogio_de_la_locura (Fecha de acceso): 15 de agosto 2020).

\section{CORRESPONDENCIA:}

Jesús Chirinos-Cáceres

jesus.chirinos@upch.pe

Fecha de recepción: 16-08-2020.

Fecha de aceptación: 24-08-2020. 